PREVALENCE AND PSYCHOLOGICAL CORRELATES OF POSTNATAL DEPRESSION IN RURAL TAIWANESE WOMEN

AUTHORS:

Ying Tsao RN PhD

Associate Professor, Department of Nursing, Tzu-Chi

University, Taiwan

(E-mail: a84016ying@hotmail.com)

Debra K Creedy RN PhD

Professor, Griffith Health Institute Griffith University, Australia

(E-mail: d.creedy@griffith.edu.au)

Jenny Gamble RM PhD

Professor, School of Nursing and Midwifery, Griffith Health Institute, Griffith

University, Australia.

(E-mail: j.gamble@griffith.edu.au)

CORRESPONDING AUTHOR

Dr Ying Tsao

Associate Professor, Department of Nursing, Tzu-Chi University, Taiwan

Postal Address: No. 701, Zhongyang Rd., Sec .3, Hualien, 97004 Taiwan

Phone: 886-3-8565301 Ext. 2231

Fax: 886-3-8574767

Email: a84016ying@hotmail.com; s2178593@mail.tcu.edu.tw 


\title{
PREVALENCE AND PSYCHOLOGICAL CORRELATES OF POSTNATAL DEPRESSION IN
}

\section{RURAL TAIWANESE WOMEN}

\begin{abstract}
This descriptive longitudinal cohort study investigated the prevalence and psychological risk factors for depression in new mothers. Expectant mothers $(n=236)$ were recruited through antenatal clinics in Pingtung County, southern Taiwan and completed a set of standardized measures including the Edinburgh Postpartum Depression Scale (EPDS), Rosenberg Self-Esteem Scale (RSE), Depression Anxiety Stress Scale (DASS-21), and General Health Questionnaire (GHQ-12). At 6 weeks postpartum, 162 participants (69\% retention) provided obstetric details, and repeated the measures. The estimated prevalence of probable depression (score of 13 or higher) was $17.3 \%$ before birth and $24.1 \%$ after birth. Women with high antenatal EPDS scores had an increased risk of postnatal depression. EPDS scores were correlated with DASS-depression $(r=.60, p<.01)$; Anxiety $(r=.59, p<.01)$ and Stress $(r=.57, p<.01)$, general health $(r=.73, p<.01)$; and self-esteem $(r=-.33, p$ $<.01)$. The hierarchical regression analysis yielded an adjusted $R^{2}$ of .70 , indicating a relatively strong association between these variables and postnatal depressive symptoms. Rural Taiwanese women are less likely to have early detection of PND risk factors and timely provision of relevant interventions. Routine screening conducted during pregnancy and postpartum are strongly recommended and appropriate intervention needs to be provided.
\end{abstract}

Keywords: Pregnancy, antenatal depression, postnatal depression, prevalence, predictors, general health, self esteem 
Postnatal depression (PND) is a common, psychological disorder among childbearing women, but there are relatively few studies of PND prevalence in women living in Asian countries. In the eastern Asian region (e.g., China, Taiwan, Hong Kong, Japan, Korea), the reported prevalence for PND ranges from almost $0 \%$ to over $60 \%$ in studies published in English (Halbreich \& Karkun, 2006). An overall weighted prevalence rate of $27.9 \%$ in Asian women has been reported (Roomruangwong \& Epperson, 2011). In Taiwan, PND prevalence has been estimated as being between $10.3 \%$ and $73.3 \%$ (as shown in Table 1), and is a significant problem. Participants, however, tended to be recruited from urban areas and less is known about the experiences of women living in rural communities. We undertook a descriptive, longitudinal, cohort study to investigate the prevalence and psychological risk factors for depression in new mothers living in rural southern Taiwan.

Over the past decades, researchers have aimed to identify possible predictors of PND. Although no clear cause of PND exists, reviews of studies involving Asian women have identified possible risk factors including biological and obstetric (e.g., mode of birth, unplanned pregnancy, baby weight, and gender), psychiatric (e.g., antenatal depression and anxiety, previous psychiatric history), psychological (e.g., poor self-image, vulnerable personality), and socio-demographic factors (e.g., stressful life events, childcare stress, social support, marital relationship, socioeconomic status, and recency of immigration) (Klainin \& Arthur, 2009; Roomruangwong \& Epperson, 2011). Early identification of risk factors and prevention strategies for PND is crucial because the adverse consequences of PND not only affect mothers, but can also adversely affect the health and well-being of their children and wider family (Dennis \& Creedy, 2007).

In Taiwan, studies related to PND have been restricted to targeted populations living in the central metropolis or urban areas such as Taipei, Taichung and Kaohsiung. 
These large cities are located on the west coast of Taiwan, and are more prosperous and technologically advanced than other areas. In particular, Taipei, the capital of Taiwan, is located in the northern part of the island and has extensive health-care resources. The characteristics and experiences of childbearing women in rural areas differ from their urban counterparts and socioeconomic disadvantage is common among rural women (Bloom, Bullock, \& Parsons, 2012). Some researchers have suggested an association between limited access to health care services and poorer health outcomes (Auger, Authier, Martinez, \& Daniel, 2009). Accordingly, the use of samples with different demographic and psychosocial characteristics may lead to a better understanding of prevalence estimates and associated risk factors of depression in rural women.

\section{METHODS}

\section{Design}

A descriptive longitudinal cohort study was used.

Participants

The inclusion criteria were as follows: women of Mainlander or Hoklos extraction, at least 18 years of age, and expected to give birth to a live, singleton baby. Women who were immigrants, had current or past mental health problems, anticipated a stillbirth, late neonatal death, or live births with a congenital anomaly were all excluded from this study.

\section{Measures}

Standardized self-report measures were used to assess maternal emotional state during both the antenatal and postnatal periods. The survey form was translated and back-translated into Taiwanese using processes suggested by Haddad, Shotar, Younger, Alzyoud, and Bouhaidar (2011).

Demographic and obstetric characteristics 
Women answered several questions about their age, education, occupation, monthly family income, and marital status. In addition, items assessing maternal feelings towards the pregnancy, parity, mode of birth, baby weight, and gender were included.

The ten item Edinburgh Postnatal Depression Scale (EPDS) was used to measure maternal depressive symptoms (Cox, Holden, \& Sagovsky, 1987). Respondents use a four point likert scale of $0=$ no, not at all to $3=$ As much as I always have to rate how they felt in the past week. There is a possible range from 0 to 30 with higher scores implying greater distress. Scores of 13 or more are highly sensitive for correctly identifying true cases of depression (Sit \& Wisner, 2009) and was applied the present study.

The Depression Anxiety Stress Scale (DASS-21) provides a measure of depression, physical arousal, and psychological tension and agitation symptoms, has been used in maternal-child health settings (Mohammad, Gamble, \& Creedy, 2011), and is easy to interpret. Using a 4-point Likert scale, ranging from 0 (Did not apply to me at all) to 3 (Applied to me very much, or most of the time), participants respond according to their wellbeing for the past 7 days. Scores for the three subscales of DASS-21 are summed and evaluated as per the severity-rating index (Lovibond \& Lovibond, 1995). Internal consistencies (Cronbach's alphas) are acceptable for all subscales (depression $\alpha=0.84$, anxiety $\alpha=0.77$, stress $\alpha=0.86$ ) (Miller, Pallant, $\&$ Negri, 2006).

Rosenberg Self-Esteem Scale (RSE) contains 10 items measuring personal worth, self-confidence, self-satisfaction, self-respect, and self-depression. Items are answered on a four-point scale, ranging from 1 (strongly agree) to 4 (strongly disagree). The scoring for some items, such as $2,5,6,8,9$, are reversed to minimize response bias. There is a possible range from 10 to 40 , with higher scores reflecting 
higher self-esteem. The RSE is valid and reliable, with a reported Cronbach's alpha ranging from .45 to .90 (Huang \& Dong, 2012). Kuster and Orth (2013) indicated that feelings of self-esteem are relatively stable, even across very long periods, and that self-esteem is a trait-like characteristic. Accordingly, in the present study, the RSE was only administered to participants during pregnancy.

General Health Questionnaire (GHQ-12) is used to detect psychiatric morbidity among medical outpatients (Goldberg, 1978). Ip and Martin (2006) reported that the GHQ-12 has been translated into numerous languages, and found to be a valid, reliable and sensitive scale for use with Chinese mothers. In the present study, items on the GHQ-12 were rated using a 4 point Likert style (0-1-2-3). There is a possible score range from 0 to 36 . Scores above 15 are evidence of distress, and scores above 20 suggest severe problems and psychological distress (Shelton \& Herrick, 2009).

Procedure

Between January and July 2008, women in their last trimester of pregnancy and attending an antenatal appointment (in one of four regional hospitals in Pingtung) were asked by clinic staff if they wished to participate in the study. Women were then approached by the researcher who provided information, assessed for eligibility, obtained informed consent, and asked them to complete four standardized psychological measures (EPDS, RSE, DASS-21, and GHQ-12). At around 6 weeks postpartum, all recruited women were followed up whilst attending their postnatal appointment or by phone. Women who did not respond to calls were deemed to be lost to follow-up. All procedures and the informed consent process employed were approved by the Human Research Ethics Committee of Griffith University and the relevant hospital committees in Taiwan.

Statistical analysis

The statistical analyzes were carried out with SPSS computer software (version 
12.0). All demographic and obstetric variables were analyzed as categorical data. Means, standard deviations, and percentages were calculated for descriptive data. Differences between scores from various measures were evaluated by paired t-test. Pearson's coefficients were calculated for correlations between continuous predictor variables (DASS-21, RSE, GHQ-12) and maternal depressive symptoms (EPDS). Significant predictors of PND were identified with hierarchical multiple regression analyses. Overall, odds radios (OR) are presented with $95 \%$ confidence intervals $(\mathrm{Cl})$ and statistical significance was defined as two-tailed $p$ values using a significance level of .05 .

\section{RESULTS}

\section{Participant characteristics}

A total of 236 women participated and provided antenatal information. Of these, 162 women were followed-up at around 6-weeks postnatal resulting in a 69\% response rate (days after birth: Mean $=44.2 ;$ S.D. $=19.3$ ). As shown in Table 2, results of the comparative analysis indicated three significant differences between the 162 women who completed the study and those lost to follow-up $(n=74)$. The 74 women lost to follow-up were significantly more likely to list their occupation as a housewife $\left(\chi^{2}=.01, d f=1, p=.02\right.$, Fischer's exact test), be younger (mean $=27.1$ vs. $29.4 ; t=$ $-3.74 ; p<.01$ ), and have fewer months of marriage (mean $=19.0$ vs. $32.4 ; t=-3.06 ; p$ $<.01)$ than other respondents.

Among the 162 participants, the mean age was $29.4(S D=4.42)$ years. About one-half of the women $(51.6 \%, n=83$ ) possessed a high school diploma and $42.2 \%$ ( $=68)$ had obtained a college diploma or university degree. The majority of participants (93.8\%) were married and nearly all women were living with the baby's father

In regards to obstetric data, more than half were first-time mothers, over 
one-third had one child and 7 percent $(n=12)$ had two or more children. Sixty percent $(n=98)$ of participants had a vaginal birth and nearly $40 \%(n=64)$ had a caesarean section, which was much higher than $15 \%$ recommended by the World Health Organization (1985). Of the babies born, $56 \%$ were boys, and $44 \%$ were girls. The proportion of neonates weighing less than 2500 grams, defined as low birth weight infants, was approximately $4.3 \%(n=7)$ in the sample. The majority of babies (70\%) weighed $3000 \mathrm{~g}$ or more.

Maternal psychological distress

Of the 162 participants completing both Time 1 and Time 2 surveys, the EPDS mean score was 8.30 (SD $=4.83$, range $0 \sim 24)$ during pregnancy, and $9.09(S D=5.60$, range $0 \sim 24)$ at 6 weeks postpartum. Twenty-eight women had probable antenatal depression and 39 had probable postnatal depression. Accordingly, the prevalence of probable depression in these women rose from $17.3 \%$ (28/162) before birth to $24.1 \%$ (39/162) postpartum. Of the 28 women reporting symptoms of probable depression before birth, 17 (60.7\%) were still depressed at 6 weeks postpartum. A moderate yet significant positive correlation $(r=.51, p<.01)$ was found between women's EPDS scores during pregnancy and postpartum.

As shown in Table 3, the mean DASS-21 depression score for pregnant rural Taiwanese women was 3.57 (SD = 5.36), which slightly increased after birth (mean = 4.74, SD $=5.89)$. Ten women (6.2\%) scored above mild on the DASS-21 depression subscale (i.e., > 13) in pregnancy, and five were still experiencing significant levels of depression after birth. However, a further 13 women who scored within the normal range on the DASS-21 depression subscale (i.e., 0 9) during pregnancy were found to score above mild (i.e., > 13) after birth. The correlation between women's scores on the EPDS and DASS-depression was significant $(r=.60, p<.01)$ (Table 4).

During pregnancy, the women's mean score for the DASS anxiety subscale was 
$6.32(S D=6.62)$, which was very similar to the mean score $($ mean $=6.31, S D=6.12)$ in the early postpartum. Pregnant women who had higher DASS-anxiety subscale scores tended to report more anxiety after childbirth $(r=.48, p<.01)$. Moreover, a significant correlation was observed between women's DASS-21 anxiety subscale scores and EPDS scores $(r=.59, p<.01)$. Suspected depressed women (EPDS scores $\geq$ 13), either during pregnancy or postpartum, had higher anxiety than undetected depressed women indicating that anxiety and depression often co-exist.

Among participants, the level of reported stress (on the DASS stress scale) increased from pregnancy to 6 weeks postpartum (mean $=6.98$ vs. 9.38). Also, women with high EPDS scores had elevated DASS-21 stress scores. There was a significant positive correlation between EPDS and DASS-21 stress scores, both during pregnancy $(r=.57 p<.01)$ and after childbirth $(r=.59, p<.01)$.

\section{Maternal GHQ-12}

Participants' mean score on the GHQ-12 was $8.54(\mathrm{SD}=5.72)$ before birth, and $10.25(\mathrm{SD}=6.27)$ after birth. Twenty-three pregnant women (14.2\%) scored above 15 and 44 postnatal mothers (27.2\%) scored above 15 , revealing a proportional increase of postnatal mothers with psychological disturbance or distress after giving birth. GHQ-12 scores were significantly associated with EPDS scores, both during pregnancy $(r=.73 p<.01)$ and after childbirth $(r=.82, p<.01)$.

\section{Maternal self-esteem}

The mean RSE score for this sample was 20.14 (SD = 3.48). Using the RSE scores, participants were categorized into low $\left(n=7\right.$, mean $=12.71, S D=1.50$, range $\left.10^{\sim} 14\right)$, medium $\left(n=144\right.$, mean $=19.95, S D=2.57$, range $\left.15^{25}\right)$, and high self-esteem groups $\left(n=11\right.$, mean $=27.36, S D=1.43$, range $\left.26^{\sim 30}\right)$. Suspected depressed women reported an RSE mean score of $18.07(S D=3.49)$, while non-depressed women had a mean score of $20.57(S D=3.33)$ prior to childbirth. There was a negative and 
statistically significant association between RSE and EPDS scores $(r=-.33, p<.01)$. Taiwanese rural women reporting low RSE were more likely to be vulnerable to depression.

Factors associated with postnatal depression symptoms

Bivariate analysis showed significant associations with postnatal depressive symptoms (EPDS) for maternal antenatal depressive symptoms, psychological distress (i.e., GHQ-12, and three subscales of DASS-21) and self-esteem (RSE). Participants' socio-demographic and obstetric factors (i.e., maternal age, education, occupation, marital status, parity, and infant details) were not predictive of higher depression scores. To test the unique association of predictive factors with depressive symptoms, all significant predictors were included in hierarchical regression analyses. Assumptions for regression analyses (i.e., normality, linearity, and homoscedasticity of residuals) were conducted, and no violations were found among the data.

Women's antenatal RSE scores negatively predicted depressed mood after birth, and accounted for $15 \%$ of the variance in the first step. In step 2, antenatal EPDS scores added an additional $16 \%$ of the variance. In step 3, GHQ-12 scores resulted in a significant increment in $R^{2}$ and explained 39\% of the variance in EPDS scores. Overall, the regression equation yielded an adjusted $R^{2}$ of .70 , indicating a relatively strong association between these variables and postnatal depressive symptoms (Table 5).

\section{DISCUSSION}

This study found a high rate of psychological distress among rural Taiwanese women. While $69 \%$ of participants were followed at 6 weeks postpartum, younger women in a housewife role with less duration of marriage were lost. Younger age, low occupation status and less stable marital relationship have been identified as possible risk factors for depression in previous studies with Asian women 
(Roomruangwong \& Epperson, 2011). It is likely therefore that women lost to follow-up in the current study were at greater risk of depression than women retained in the study. The reported prevalence rate may be an under-estimation of depression and psychological distress amongst these rural women.

There are many challenges to conducting research with rural women, even in small industrialized countries such as Taiwan where distances are small but mountainous geography can create isolation. In Taiwan, there is no fixed schedule for postnatal visits and the approach to postnatal assessment varies. Although one postnatal visit at 6 weeks postpartum is recommended under the National Taiwanese Health Insurance system (Chien, Tai, Hwang, \& Huang, 2009), many rural women did not attend. Non-attendance may be due to the costs associated with travel, cost of transport, and loss of income from time away from work.

Researchers have found the prevalence of depression progressively decreases toward the end of a pregnancy (Bergink et al., 2011). However, at around 4 weeks postpartum, women have the highest risk for depressive symptoms (Mori et al., 2011) and between 8 and 12 weeks have an increased risk for major depressive disorder. It is not surprising that estimates of PND are found to be greater in studies with a wider time window than a narrow one (e.g., first four weeks) (Halbreich \& Karkun, 2006). Accordingly, the time periods in the current study were extended to the third trimester of pregnancy, and 6 weeks postpartum. It was an attempt to explicitly capture the variability in prevalence rates of antenatal and postnatal depression.

A strength of the current study is that a quantitative longitudinal study design was used rather than a cross-sectional survey (Chen, Kuo, Chou, \& Chen, 2007; Chien, Tai, Ko, Huang, \& Sheu, 2006). This design enabled us to make credible claims as to the assessment of individual changes and differences among the mothers.

The study design also enabled us to address some fundamental considerations 
associated with the use of standardized measures to identify women experiencing PND. The use of the EPDS allowed for comparisons amongst different samples as it is the most frequently used scale followed by the Beck Depression Inventory (BDI-II), and the Centre of Epidemiologic Study-Depression (CES-D) in Taiwanese studies. A statistical validation of the EPDS with a structured psychiatric interview was recently confirmed in Taiwan (Su et al., 2007). Other researchers have found that the EPDS has better specificity than the BDI-II and yields less false-positive results, while providing the same sensitivity. The BDI-II contains more somatic items than the EPDS, and may not be the best solution when screening for mood disorders in pregnant/postpartum women (Curžik D \& Begić, 2012).

The prevalence rate of maternal depression among these rural Taiwanese women was about $17 \%$, which increased to $24.1 \%$ at 6 weeks following childbirth. These prevalence rates are higher, but comparable to, rates reported by authors in related Taiwan studies (Heh, Huang, Ho, Fu, \& Wang, 2008; Huang \& Mathers, 2001; Teng et al., 2005). It is possible that the higher PND prevalence in the current sample reflects women's response to their specific situation (i.e., socioeconomic adversity or exposure to adverse experiences). Rural (Pingtung) residents are more likely, in comparison with urban people, to experience barriers in accessing appropriate information and healthcare services because of geographic isolation, centralization of services, and livelihood constraints. Despite the discrepant findings between studies, there is evidence that the PND prevalence among women of childbearing age in rural Taiwan is as high or even higher than that of Western populations. Postnatal depression is a significant health issue nationally and internationally.

In the present study, participants who were not employed outside the home tended to have lower self-esteem. Those who reported low self-esteem also tended to report higher levels of depression. Researchers have shown conflicting 
results as to whether women's employment may influence their health. For some women, employment increases their risk for physical and mental health problems as a result of exposure to work stress or overload. For others, employment may result in increased income, better access to health care, or an increased sense of well-being. In a survey of 270 postnatal Taiwanese women regarding work status and maternal mental well-being, Chen (2001) found that unemployed mothers were at significant increased risk for depression in terms of prevalence and severity as well as lower self-esteem. Therefore, it seems more likely that employment has positive benefits for mental health and enhancing the self-esteem of women in this culture.

We found the psychological risk factors for depression following childbirth included depression during pregnancy, low self-esteem (RSE), and the presence of psychological distress (DASS-21) and poor general health (GHQ-12). The predictive regression model for postnatal depressive symptoms (EPDS) in these Taiwanese women accounted for $70 \%$ of variance. We confirmed that women who experienced significant levels of depressive symptoms before birth were vulnerable to subsequent postnatal depressive symptoms. Pregnant women with low self-esteem had a higher risk of postnatal depression. Furthermore, postnatal women with high GHQ-12 scores were more likely to report elevated scores on the EPDS and this finding is similar to those reported previously by other authors (Lee et al., 2000; Navarro et al., 2007).

\section{CONCLUSIONS AND IMPLICATIONS}

Relatively high rates of reported depressive symptoms among childbearing women in rural Taiwanese women were identified. In light of these results, routine psychological and risk assessment of expectant mothers for depression is strongly recommended in clinical practice. Facilities/services are required to regularly monitor women's health status both physically and psychologically. Given the high rate of depressive symptoms identified, it will be necessary for health care providers (such 
as nurse practitioners or midwives) who care for childbearing women to be familiar with signs of depression during pregnancy and in the transition to motherhood. Health professionals, especially those working in rural or disadvantaged areas, should receive special training and achieve competency in psychosocial assessment, preventative strategies and interventions to care for women experiencing depressive symptoms.

\section{ACKNOWLEDGEMENTS}

The authors extend their deep appreciation to the mothers for taking part in this study and the four participating hospitals for enabling the authors to collect data directly.

\section{REFERENCES}

Affonso, D. D., De, A. K., Horowitz, J. A., \& Mayberry, L. J. (2000). An international study exploring levels of postpartum depressive symptomatology. Journal of Psychosomatic Research, 49(3), 207-216.

Auger, N., Authier, M.-A., Martinez, J., \& Daniel, M. (2009). The association between rural-urban continuum, maternal education and adverse birth outcomes in Québec, Canada. The Journal of Rural Health, 25(4), 342-351.

Bergink, V., Kooistra, L., Lambregtse-van den Berg, M. P., Wijnen, H., Bunevicius, R., van Baar, A., et al. (2011). Validation of the Edinburgh Depression Scale during pregnancy. Journal of Psychosomatic Research, 70(4), 385-389.

Bloom, T. L., Bullock, L. F. C., \& Parsons, L. (2012). Rural pregnant women's stressors and priorities for stress reduction. Issues in Mental Health Nursing, 33(12), 813-819.

Chen, C.-M., Kuo, S.-F., Chou, Y.-H., \& Chen, H.-C. (2007). Postpartum Taiwanese women: Their postpartum depression, social support and health-promoting lifestyle profiles. Journal of Clinical Nursing, 16(8), 1550-1560.

Chen, C. H. (2001). Association of work status and mental well-being in new mothers. Kaohsiung Journal of Medical Sciences, 17, 570-575.

Chien, L.-Y., Tai, C.-J., Hwang, F.-M., \& Huang, C.-M. (2009). Postpartum physical symptoms and depressive symptomatology at 1 month and 1 year after delivery: A longitudinal questionnaire survey. International Journal of Nursing Studies, 46(9), 1201-1208.

Chien, L.-Y., Tai, C.-J., Ko, Y.-L., Huang, C.-H., \& Sheu, S.-J. (2006). Adherence to "Doing-the-month" practices is associated with fewer physical and depressive 
symptoms among postpartum women in Taiwan. Research in Nursing \& Health, 29(5), 374-383.

Cox, J., Holden, J., \& Sagovsky, R. (1987). Detection of postnatal depression: Development of the 10-item Edinburgh Postnatal Depression Scale. British Journal of Psychiatry, 150, 782-786.

Curžik D, \& Begić, N. J. (2012). The utility of BDI-II in assessment of pre- and postpartum depression symptoms and their relation to labor pain. Psychiatr Danub, 24(2), 167-174.

Dennis, C.-L., \& Creedy, D. (2007). Psychosocial and psychological interventions for preventing postpartum depression. Cochrane Database of Systematic Reviews, (4), 1-55.

Gibson, J., McKenzie-McHarg, K., Shakespeare, J., Price, J., \& Gray, R. (2009). A systematic review of studies validating the Edinburgh Postnatal Depression Scale in antepartum and postpartum women. Acta Psychiatrica Scandinavica, 119(5), 350-364.

Goldberg, D. P. (1978). Manual of the general health questionnaire. London: NFER.

Haddad, L. G., Shotar, A., Younger, J. B., Alzyoud, S., \& Bouhaidar, C. M. (2011). Screening for domestic violence in Jordan: Validation of an Arabic version of a domestic violence against women questionnaire. International Journal of Women's Health, 3, 79-86.

Halbreich, U., \& Karkun, S. (2006). Cross-cultural and social diversity of prevalence of postpartum depression and depressive symptoms. Journal of Affective Disorders, 91(2), 97-111.

Heh, S.-S., Huang, L.-H., Ho, S.-M., Fu, Y.-Y., \& Wang, L.-L. (2008). Effectiveness of an exercise support program in reducing the severity of postnatal depression in Taiwanese women. Birth, 35(1), 60-65.

Huang, C., \& Dong, N. (2012). Factor structures of the Rosenberg Self-Esteem Scale: A meta-analysis of pattern matrices. European Journal of Psychological Assessment, 28(2), 132-138.

Huang, Y.-C., \& Mathers, N. (2001). Postnatal depression - biological or cultural? A comparative study of postnatal women in the UK and Taiwan. Journal of Advanced Nursing, 33(3), 279-287.

Ip, W. Y., \& Martin, C. R. (2006). Factor structure of the Chinese version of the 12-item General Health Questionnaire (GHQ-12) in pregnancy. Journal of Reproductive \& Infant Psychology, 24(2), 87-98.

Klainin, P., \& Arthur, D. G. (2009). Postpartum depression in Asian cultures: A literature review. International Journal of Nursing Studies, 46(10), 1355-1373.

Kuster, F., \& Orth, U. (2013). The long-term stability of self-esteem: Its time-dependent decay and nonzero asymptote. Personality and Social Psychology 
Bulletin, 39(5), 677-690.

Lee, D. T. S., Yip, A. S. K., Chiu, H. F. K., \& Chung, T. K. H. (2000). Screening for postnatal depression using the double-test strategy. Psychosomatic Medicine, 62(2), 258-263.

Lovibond, P. F., \& Lovibond, S. H. (1995). The structure of negative emotional states: comparison of the Depression Anxiety Stress Scales (DASS) with the Beck Depression and Anxiety Inventories. Behaviour Research \& Therapy, 33(3), 335-343.

Miller, R. L., Pallant, J. F., \& Negri, L. M. (2006). Anxiety and stress in the postpartum: Is there more to postnatal distress than depression? BMC Psychiatry 6(1), 1-11.

Mohammad, K. I., Gamble, J., \& Creedy, D. K. (2011). Prevalence and factors associated with the development of antenatal and postnatal depression among Jordanian women. Midwifery, 27(6), e238-e245.

Mori, T., Tsuchiya, K. J., Matsumoto, K., Suzuki, K., Mori, N., \& Takei, N. (2011). Psychosocial risk factors for postpartum depression and their relation to timing of onset: The Hamamatsu Birth Cohort (HBC) Study. Journal of Affective Disorders, 135(1), 341-346.

Navarro, P., Ascaso, C., Garcia-Esteve, L., Aguado, J., Torres, A., \& Martín-Santos, R. (2007). Postnatal psychiatric morbidity: A validation study of the GHQ-12 and the EPDS as screening tools. General Hospital Psychiatry, 29(1), 1-7.

Roomruangwong, C., \& Epperson, C. N. (2011). Perinatal depression in Asian women: Prevalence, associated factors, and cultural aspects. Asian Biomedicine, 5(2), 179-193.

Shelton, N. J., \& Herrick, K. G. (2009). Comparison of scoring methods and thresholds of the General Health Questionnaire-12 with the Edinburgh Postnatal Depression Scale in English women. Public Health, 123(12), 789-793.

Sit, D. K. Y., \& Wisner, K. L. (2009). Identification of Postpartum Depression. Clinical Obstetrics and Gynecology, 52(3), 456-468

Su, K.-P., Chiu, T.-H., Huang, C.-L., Ho, M., Lee, C.-C., Wu, P.-L., et al. (2007). Different cutoff points for different trimesters? The use of Edinburgh Postnatal Depression Scale and Beck Depression Inventory to screen for depression in pregnant Taiwanese women. General Hospital Psychiatry, 29(5), 436-441.

Teng, H.-W., Hsu, C.-S., Shih, S.-M., Lu, M.-L., Pan, J.-J., \& Shen, W. W. (2005). Screening postpartum depression with the Taiwanese version of the Edinburgh Postnatal Depression Scale. Comprehensive Psychiatry, 46(4), 261-265.

Wang, S.-Y., \& Chen, C.-H. (2006). Psychosocial health of Taiwanese postnatal husbands and wives. Journal of Psychosomatic Research, 60(3), 303-307.

Wang, S.-Y., Jiang, X.-Y., Jan, W.-C., \& Chen, C.-H. (2003). A comparative study of postnatal depression and its predictors in Taiwan and mainland China. American 
Journal of Obstetrics and Gynecology, 189(5), 1407-1412. 


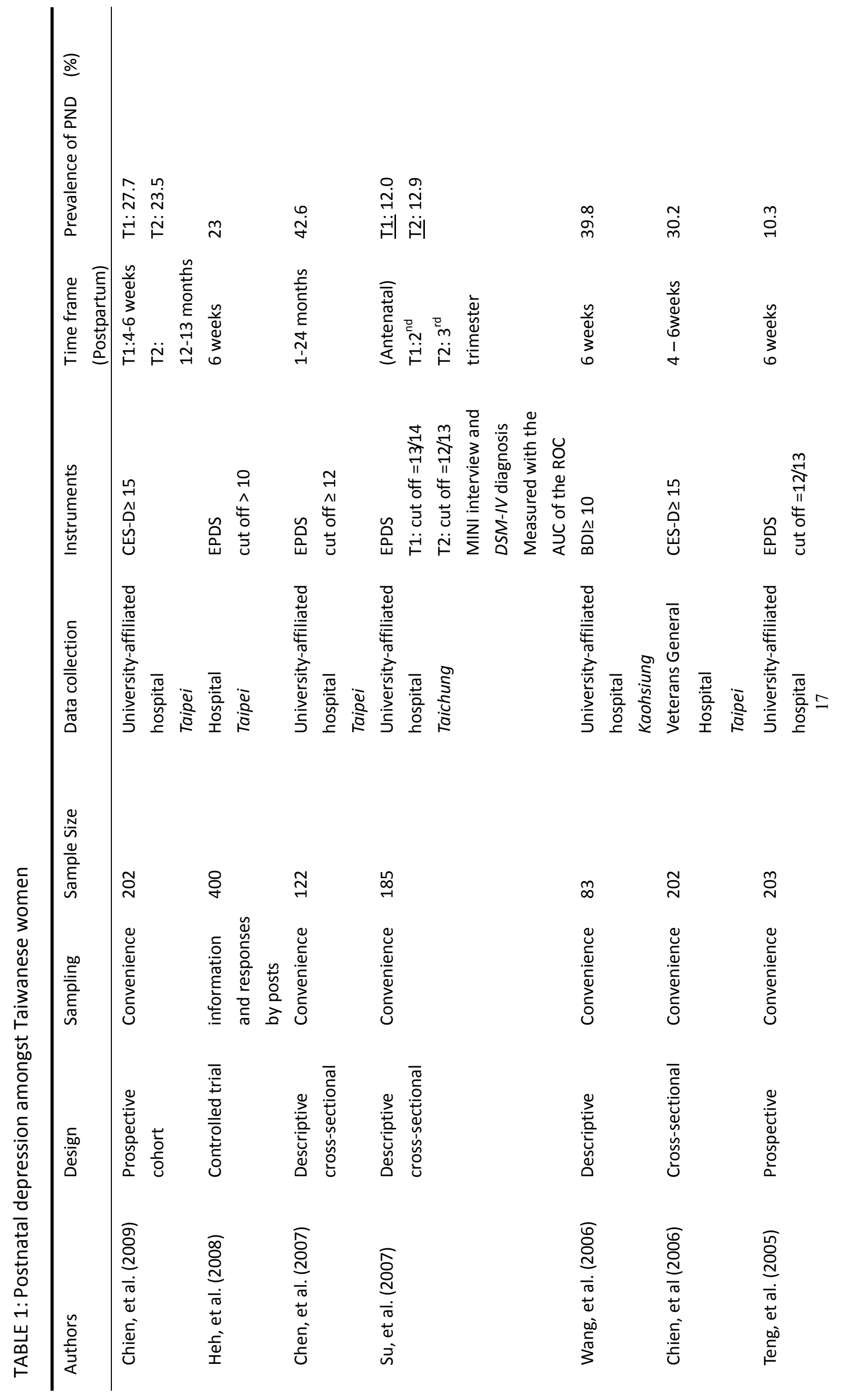




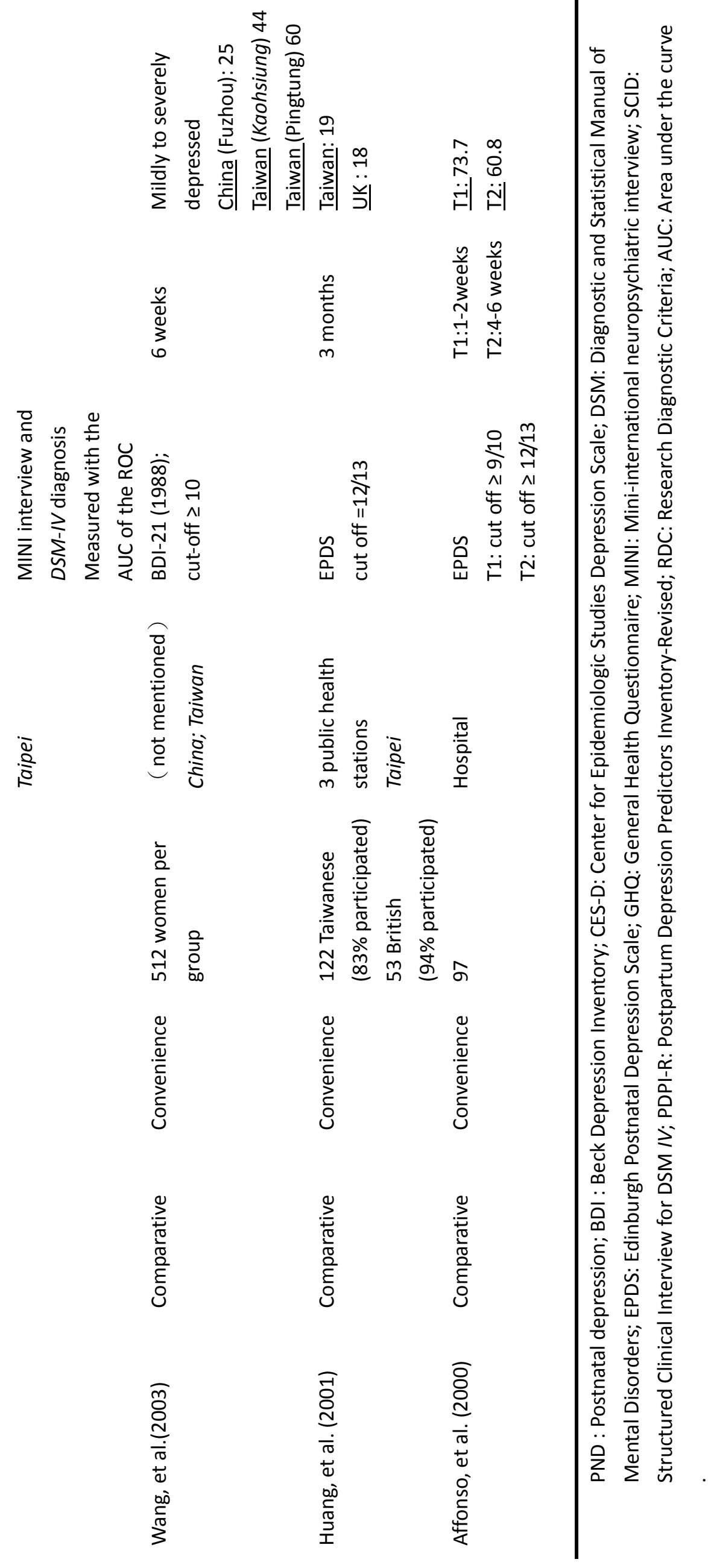


TABLE 2: Demographic and obstetric characteristics of rural Taiwanese women

\begin{tabular}{|c|c|c|c|c|}
\hline $\begin{array}{l}\text { Variables } \\
\text { Demographic \& Obstetric }\end{array}$ & $\begin{array}{l}\text { Total (\%) } \\
(n=236)\end{array}$ & $\begin{array}{l}\text { Respondents } \\
\left(n_{1}=162\right)\end{array}$ & $\begin{array}{l}\text { Lost to *FU } \\
\left(n_{2}=74\right)\end{array}$ & $p$ (2-tailed) \\
\hline Maternal age in years & 234 & 160 & 74 & $<.001 * *$ \\
\hline (1) Under 20 & $4(1.7)$ & 3 & 1 & Mean $_{1}=29.4 \mathrm{y}$ \\
\hline $20 \sim 24$ & $40(17.1)$ & 18 & 22 & $\operatorname{Mean}_{2}=27.1 \mathrm{y}$ \\
\hline (2) $25 \sim 29$ & $88(37.6)$ & 59 & 29 & \\
\hline (3) $30 \sim 34$ & 78 (33.3) & 59 & 19 & \\
\hline $35 \sim 39$ & $24(10.2)$ & 21 & 3 & \\
\hline Missing data & 2 & 2 & 0 & \\
\hline Marital status & 236 & 162 & 74 & 1.00 (ns) \\
\hline Married & $222(94.1)$ & 152 & 70 & \\
\hline $\begin{array}{l}\text { Unmarried } \\
\text { (single, divorce, separated) }\end{array}$ & $14(5.9)$ & 10 & 4 & \\
\hline $\begin{array}{l}\text { Duration of married life } \\
\text { (Months) }\end{array}$ & 206 & 145 & 61 & $<.001 * *$ \\
\hline Valid number & 206 & 145 & 61 & Mean $_{1}=32.4 \mathrm{~m}$ \\
\hline Missing data & 30 & 17 & 13 & Mean $_{2}=19.0 \mathrm{~m}$ \\
\hline Education level & 235 & 161 & 74 & \\
\hline (1) 9-year schooling & $14(6.0)$ & 10 & 4 & .07 (ns) \\
\hline (2) 12 -year school & 89 (37.9) & 83 & 36 & Mean $_{1}=14.3$ \\
\hline (3) $>12$-year schooling & $132(56.2)$ & 68 & 34 & Mean $_{2}=13.8$ \\
\hline Missing data & 1 & 1 & & \\
\hline Health insurance coverage & 236 & 162 & 74 & .30 (ns) \\
\hline One (NHI) & $89(37.7)$ & 57 & 32 & \\
\hline More than one & $147(62.3)$ & 105 & 42 & \\
\hline Occupation & 233 & 131 & 72 & $.02 *$ \\
\hline (1) Home-based & $126(54.1)$ & 79 & 47 & \\
\hline (2) Outside & $107(45.9)$ & 82 & 25 & \\
\hline Missing data & 3 & 1 & 2 & \\
\hline Gross Family Income & 220 & 153 & 67 & .62 (ns) \\
\hline$<30,000$ & $36(16.4)$ & 23 & 13 & \\
\hline $30,000 \sim 50,000$ & 93 (42.3) & 64 & 29 & \\
\hline$>50,000$ & 91 (41.4) & 66 & 25 & \\
\hline Missing data & 16 & 9 & 7 & \\
\hline Parity & 236 & 162 & 74 & .60 (ns) \\
\hline Nulliparous & $136(57.6)$ & 91 & 45 & \\
\hline Multiparous & $100(42.4)$ & 71 & 29 & \\
\hline No. of children & 236 & 162 & 74 & .57 (ns) \\
\hline None & $136(57.6)$ & 91 & 45 & \\
\hline One & $85(36.0)$ & 59 & 26 & \\
\hline More than one & $15(6.4)$ & 12 & 3 & \\
\hline Feeling toward pregnancy & 236 & 162 & 74 & .76 (ns) \\
\hline $\begin{array}{l}\text { (1) Negative (Not special, very } \\
\text { unhappy,) }\end{array}$ & $12(5.1)$ & 7 & 5 & \\
\hline (2) Mixed & $37(15.7)$ & 27 & 10 & \\
\hline (3) Pleased & $125(53.0)$ & 84 & 41 & \\
\hline (4) Overjoyed & $62(26.3)$ & 44 & 18 & \\
\hline \multicolumn{5}{|l|}{ Mode of birth } \\
\hline Normal Spontaneous & & 98 & & \\
\hline Cesarean Section & & 64 & & \\
\hline \multicolumn{5}{|l|}{ Baby gender } \\
\hline Male & & 92 & & \\
\hline Female & & 70 & & \\
\hline \multicolumn{5}{|l|}{ Neonatal weight } \\
\hline Less than $2500 \mathrm{~g}$ & & 7 & & \\
\hline $2500 \sim 3000 \mathrm{~g}$ & & 42 & & \\
\hline $3001 \sim 3500 \mathrm{~g}$ & & 85 & & \\
\hline More than $3500 \mathrm{~g}$ & & 28 & & \\
\hline
\end{tabular}

$* * \quad p<.01 ; \quad * F U$ = follow-up 


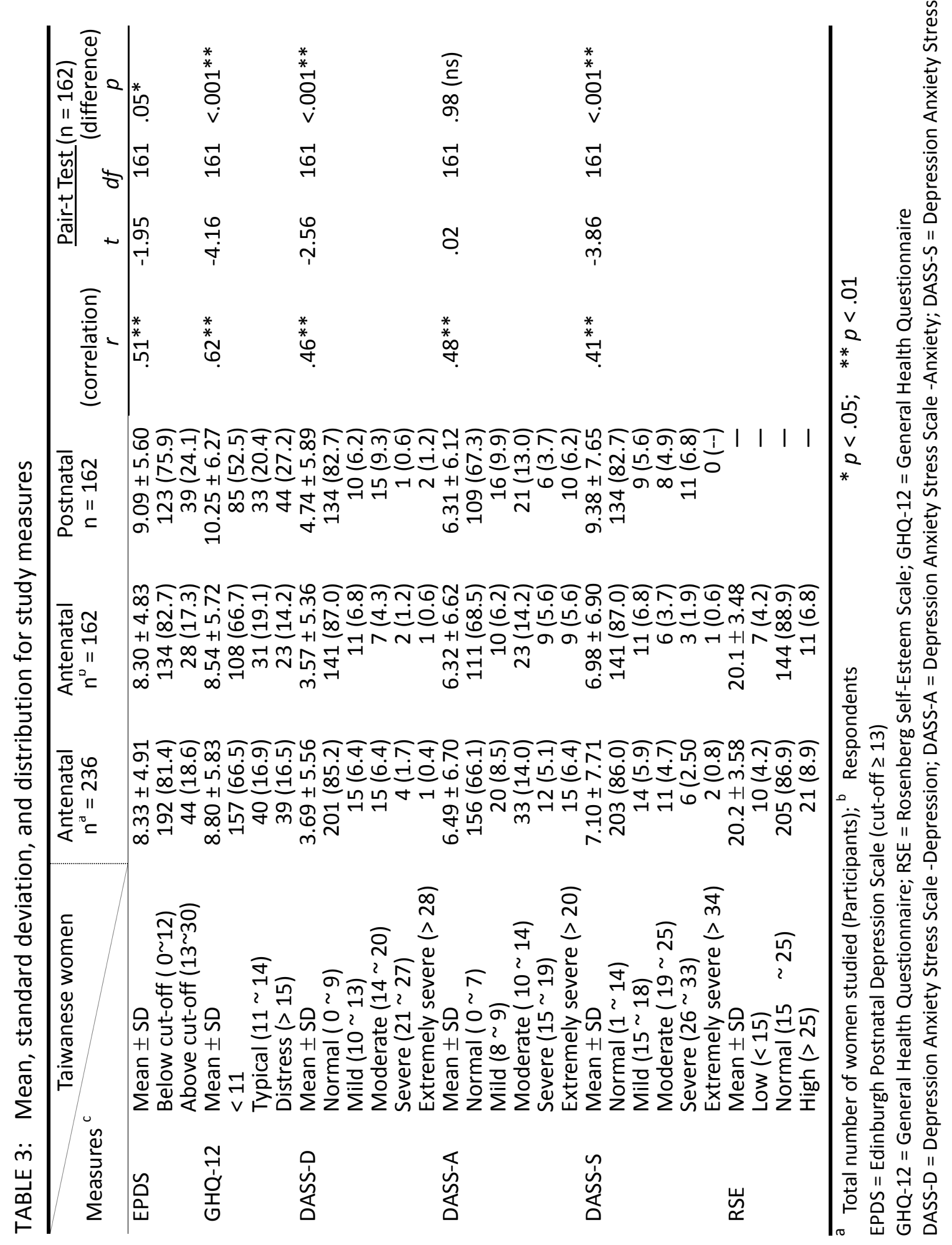




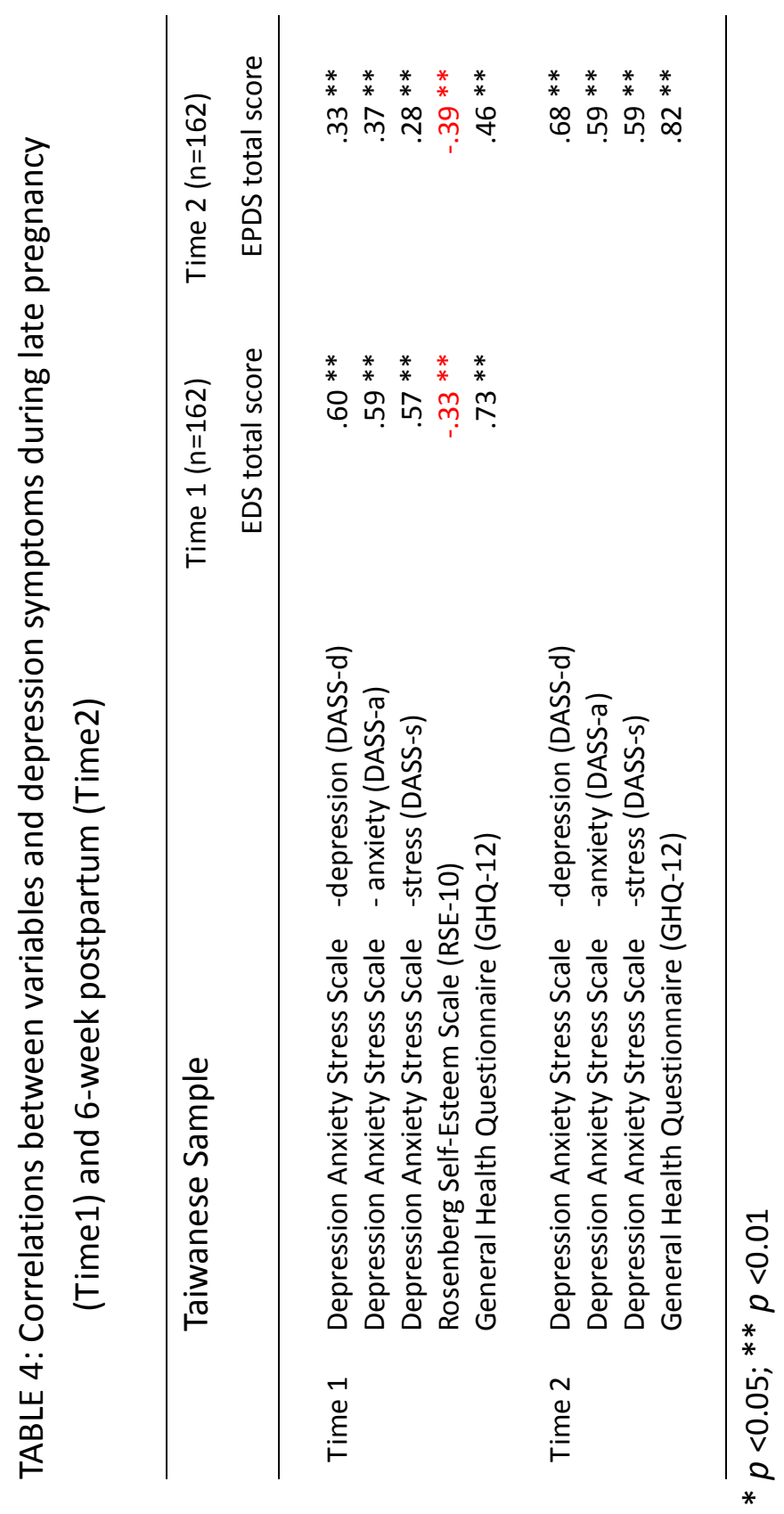




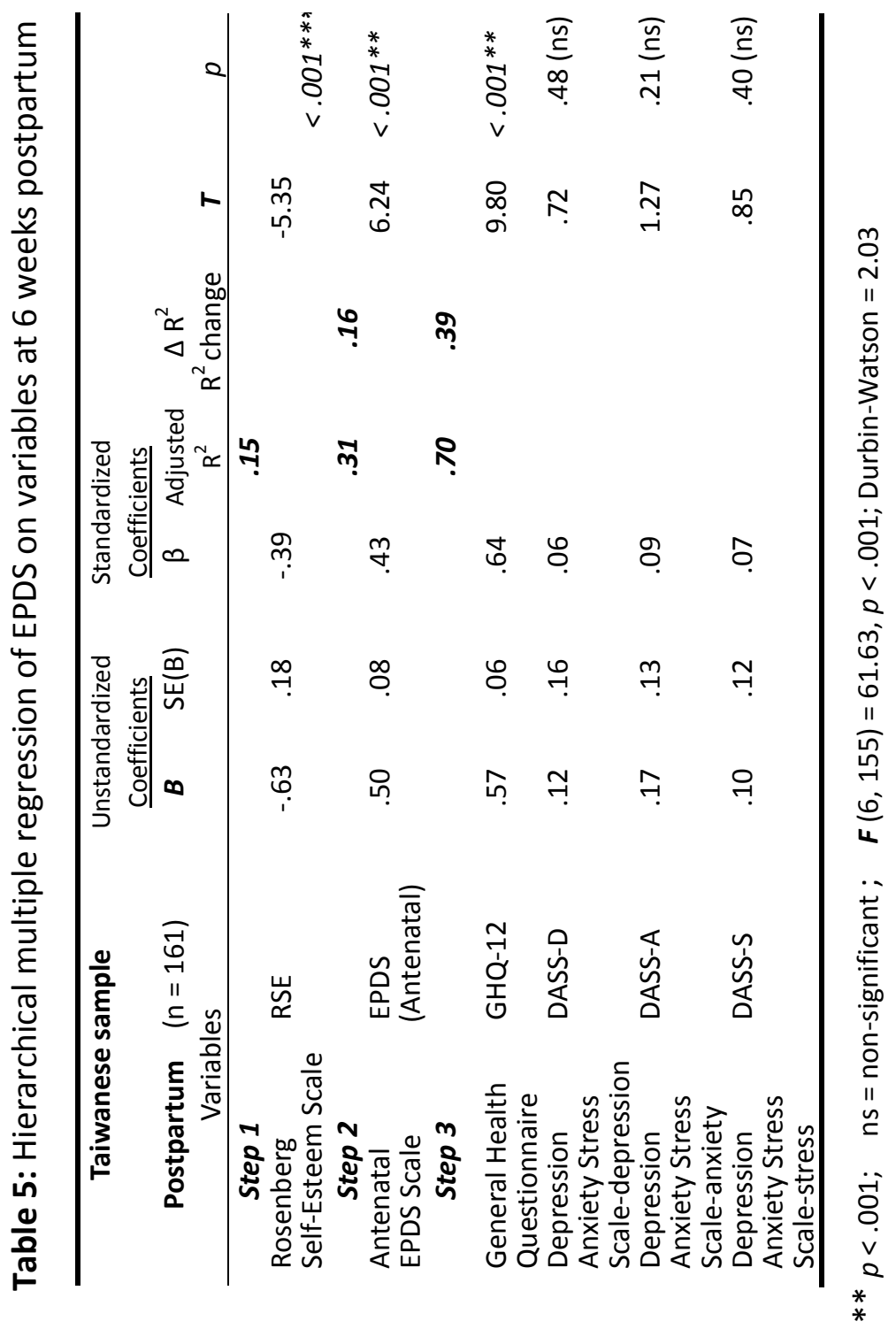

